Pure \& Appl. Chem., Vol. 58, No. 12, pp. 1553-1560, 1986.

Printed in Great Britain.

(C) 1986 IUPAC

\title{
Polymer-polymer miscibility
}

\author{
Sonja Krause \\ Department of Chemistry, Rensselaer Polytechnic Institute, Troy, IIY 12180
}

\begin{abstract}
The theoretical and practical aspects of polymer-polymer miscibility in the solid amorphous state are reviewed. The polymers include homopolymers and both random and block copolymers. Although present theoretical treatments of polymer-polymer miscibility all contain the randor mixing hypothesis and are thus not applicable to mixtures that involve specific interactions between the components, most of the observed singlephase polymer-polymer mixtures involve hydrogen-bonding or other specific interactions between the components. Even in the absence of specific interactions, the composition of a random copolymer can often be tailored to provide miscibility with a particular homopolymer. Many polymer-polymer mixtures have lower critical solution temperatures, and a small number of such mixtures have given indications of upper critical solution temperatures. The special phenomena that may be observed when other polymers are mixed with block copolymers are discussed.
\end{abstract}

\section{INTRODUCTION}

Since I have recently completed the tables on miscible polymers for the third edition of the Polymer Handbook (1), it seems appropriate to summarize present knowledge in this field at this time. This study was limited to polymers that are miscible on a segmental, submolecular scale in the solid amorphous state which may be either glassy or rubbery. A polymer which could crystallize was considered miscible with those other polymers which were miscible with its amorphous portions.

Unfortunately, the literature in this field is complicated by the interchangeable use of the terms miscibility and compatibility by many workers and by the diversity of definitions of polymer-polymer compatibility that are current in the polymer industry. In the polymer industry, a compatible polymer pair is often simply one that has desirable properties after the polymers have been mixed either as solids or in solution (with subsequent evaporation of the solvent); often, in such samples, several amorphous phases with different compositions are present. Such polymers are not miscible, but they may be referred to as compatible in the literature if there is good adhesion between the phases. Because of this confusion, I shall use only the term "miscibility" in this paper, not "compatibility."

Studies of miscibility which involve polymers are also complicated by the fact that all synthetic polymers are multicomponent systems. At the least, any sample of synthetic polymer is polydisperse with respect to (a) molecular weight; even the so-called monodisperse synthetic polymers have a measureable molecular weight distribution. Many samples are also polydisperse with respect to (b) microstruture, that is with respect to the ways in which the monomeric repeat units are inserted into the polymer chain. For example, a sample of polyvinylidene fluoride has most of the $-\mathrm{CH}_{2} \mathrm{CF}_{2}$ - repeat units in the chain added head-to-tail, $-\mathrm{CH}_{2} \mathrm{CF}_{2} \mathrm{CH}_{2} \mathrm{CF}_{2-}$, but in many cases up to about $6 \%$ of the monomer additions may have been head-to-head, $-\mathrm{CH}_{2} \mathrm{CF}_{2} \mathrm{CF}_{2} \mathrm{CH}_{2}$-; other examples include meso or racemic additions of vinyl monomers such as methyl methacrylate in the polymer chain and variable microstructure such as cis-1,4-, trans-1,4- and 1,2- addition of butadiene repeat units in polybutadiene. Many polymers will be polydisperse with respect to (c) branching, that is, number, length, and position of branches on the main polymer chain. For example, polyethylene made by different methods varies a great deal with respect to number and length of branches. Copolymers may, in addition, be polydisperse with respect to (d) composition of the different polymer molecules that comprise the sample. Random copolymers prepared by batch polymerization to high degrees of conversion of monomer to polymer may have very broad composition distributions, so broad that some samples separate into two phases with different average compositions (more about this later). Copolymers may also be polydisperse with respect to (e) sequence, that is, the sequence in which the monomers appear in each polymer molecule. He talk about copolymers as if they are either random copolymers, 
in which the different monomers are arranged randomly along the chain, or block copolymers, in which the differerent monomers are arranged as two or more pure blocks attached to each other along the chain. Not only do intermediate cases exist, but a composition distribution in a copolymer is usually accompanied by a sequence distribution.

It is well known that the solubility of a homopolymer in a solvent or in another polymer varies with the average molecular weight, the average microstructure, and with the average amount of branching of the polymer. It is also well known that the solubility of copolymers varies not only with these three factors, but also with changes in average composition and average sequence type. Experimental data that have been obtained on polymer-polymer mixtures have often been obtained on extremely polydisperse samples whose average molecular weights and other characteristics are as uncharacterized as their polydispersities. In spite of this, much is now known about polymer-polymer miscibility as a result of these experiments; I shall outline the results below.

\section{THEORETICAL TREATMENTS}

The Flory Huggins theory (2-5) of polymer solutions was the first to consider the longchain nature of high polymers, and, soon after it was proposed, Scott (6) and Tompa (7) applied it to polymer-polymer mixtures and obtained the following free energy of mixing of two polymers, $\Delta G_{\text {mix }}$ :

$$
\Delta G_{\text {mix }}=\left(R T V / V_{r}\right)\left[\left(\phi_{A} / x_{A}\right) \ln \phi_{A}+\left(\phi_{B} / x_{B}\right) \ln \phi_{B}+x_{A B} \phi_{A} \phi_{B}\right]
$$

where $V_{r}$ is a reference volume which is taken as close to the molar volume of the smallest polymer repeat unit as possible, $\phi_{A}$ and $\phi_{B}$ are the volume fractions of the two polyrners, $A$ and $B$, respectively, $x_{A}$ and $x_{B}$ are the degrees of polymerization of polymers $A$ and $B$ in terms of the reference volume $V_{r}$, respectively, and $X_{A B}$ is the interaction parameter between the two polymers. In equation 1 , the first two terms on the right hand side are conbinatorial entropy of mixing terms, calculated for a random mixture of the two polymers. Because each of these terms has a degree of polymerization, $x_{A}$ or $x_{B}$, a number greater than 100 for any high polymer, in the denominator, the entropy of mixing of two polymers is very small. The third term on the right-hand-side of equation 1 is a positive enthalpy change on mixing that was originaliy based on regular solution theory (8-10). Since even a srall positive (unfavorable) enthalpy change on mixing will outweigh the very small combinatorial entropy change on mixing in the Flory-Huggins theory, it was generally accepted for many years that most polymers should not be miscible with each other. Up to and including my review article on polymer-polymer compatibility published in 1978 (11), only about 10 percent or less of all polymer pairs investigated experimentally appeared to be miscible, as expected from the above theoretical considerations.

Since that time, many more miscible polymer pairs have been discovered. This occured because many workers have deliberately investigated those polymers in which miscibility is to be expected in the amorphous state. One way to induce miscibility is to use a polymer pair which has a negative enthalpy of mixing because of specific interactions. These specific interactions are generally hydrogen bonds but may be ion-dipole or cationanion interactions. If the specific interactions are strong enough, copolymers containing only a small percentage of mutually interacting groups have been found to be miscible.

In the absence of specific interactions, one way to find a miscible polymer pair is to choose an appropriate random copolymer as one of the polymers to be mixed. The reason for this can be found in a slight amplification of Flory-Huggins theory. If the interaction parameter in equation 1 is written in terms of Hildebrand $(8,9)$ solubility parameters, then

$$
x_{A B}=\left(V_{r} / R T\right)\left(\delta_{A}-\delta_{B}\right)^{2}
$$

where $\delta_{A}$ and $\delta_{B}$ are the Hildebrand solubility parameters for $A$ and $B$, respectively. Equation 2 shows that, when written in terms of Hildebrand solubility parameters, $X_{A B}$ is always a positive number, and since the third term on the right-hand-side of equation 1 is the enthalpy change on mixing of polymers $A$ and $B$, that the enthalpy change on mixing is also a positive number, as already mentioned. As equation 2 is written, $x_{A B}, \delta_{A}$, and $\delta_{B}$ may refer to random copolymers as well as to homopolymers. It turns out to be relatively simple to find out how to calculate the Hildebrand solubility parameters for random copolymers as long as those for the homopolymers corresponding to the monomers in the random copolymers are known. Hildebrand solubility parameters of homopolymers were discussed in reference 11.

My coworkers and I showed in 1965 (12), that when random copolymers C and D were mixed, the interaction parameter between these two randon copolymers, $X C D$, could be calculated from the homopolymer-homopolymer interaction parameters, $X_{I J}$, corresponding to the various 
monomers, I and J, of which the copolymers were composed:

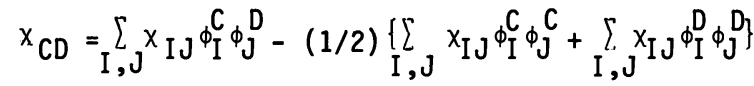

where $\phi_{I}^{C}$ refers to the volume fraction of monomer I in copolymer $C$, with similar definitions for the similar terms. The use of the same subscripts, I and J, for the monomers in copolymers $C$ and $D$ is for mathematical simplicity and does not necessarily imply that the two components contain the same monomers. Either one or both of polymers $C$ and $D$ could be homopolymers; if $C$ and $D$ are actually homopolymers $A$ and $B$, then equation 3 becomes the identity $X_{A B}=X_{A E}$. The interaction parameter for a homopolymer $A$ with a copolymer $C$ made up of volume fraction $\phi_{B}$ of monomer $B$ and volume fraction $\phi_{E}$ of monomer $E$ is then, from equation 3 , equal to

$$
x_{A C}=x_{A B} \phi_{B}^{C}+x_{A C} \phi_{E}^{C}-x_{B E} \phi_{B}^{C} \phi_{E}^{C}
$$

Equation 4 shows clearly that $X_{A C}$ is not simply a weighted average of $X_{A B}$ and $X A E$, but is less than this weighted average by a factor that depends on $X_{B E}$, the interaction parameter between the two constituents of copolymer $C$. If each of the interaction parameters in equation 4 is written in terms of Hildebrand solubility parameters as in equation 2 , then it turns out that

$$
{ }^{\delta_{C}}=\delta_{B} \phi_{B}+\delta_{E} \phi_{E}
$$

where $\delta_{C}$ is the Hildebrand solubility parameter of random copolymer $C$ and $\delta_{B}$ and $\delta_{E}$ are the Hildebrand solubility parameters of the homopolymers corresponding to monomers $B$ and $E$. Thus, the Hildebrand solublity parameter of a random copolymer is the weighted average of those of the homopolymers corresponding to its constituent monomers. Thus, also, the solubility parameter of a random copolymer is between those of the homopolymers corresponding to its constituent monomers; the exact value of ${ }^{\delta} \mathrm{C}$ also depends on the composition of the copolymer. Because of this, samples of random copolymer made up of the same monomers but with different compositions may have their solubility parameters so far apart that they will be immiscible. This may occur, as stated above, in random copolymer samples synthesized in such a way that a drift in composition, a composition distribution, occurred during the synthesis.

These ideas can also be used to find a random copolymer that should be miscible with a chosen homopolymer. Thus, when considering polymers in which specific interactions cannot occur, it is often possible to find a random copolymer made up of monomers whose solubility parameters straddle that of the homopolymer or other random copolymer with which it should be miscible. If the solubility parameters of two polymers are equal or very close to each other, then the interaction parameter between the polymers will be zero or close to zero (equation 2) and the enthalpy change of mixing will be close to zero, allowing even the small combinatorial entropy change on mixing of the Flory-Huggins theory (equation 1) to induce miscibility.

Recently, equation 3 and its consequences have been rediscovered by ten Brinke et al (13) and Paul and Barlow (14); this rediscovery has motivated some successful searches for miscible polymers in which at least one component is a random copolymer.

Flory-Huggins theory, whether applied to homopolymers or to copolymers, predicts phase diagrams with only upper critical solution temperatures. Both polymer-solvent and polymerpolymer mixtures, however, are more likely to exhibit lower critical solution temperatures than upper critical solution temperatures. Because of this, a number of authors developed corresponding states, sometimes called equation-of-state theories of polymer solutions. Flory's (15) corresponding states treatment of 1 iquid mixtures was first expanded to polymer-polymer mixtures by licllaster (16), who was able to predict both upper and lower critical solution temperatures for such mixtures. Sanchez (17) applied the corresponding states theory of Sanchez and Lacombe $(18,19)$ to polymer-polymer mixtures with similar results. These and other corresponding states theories of polymer solutions and mixtures predict negative as well as positive enthalpies of mixing, but these are not based on specific interactions; the corresponding states theories are still based on the random mixing assumption that does not allow for specific interactions. Therefore, no existing theoretical treatment is applicable to those of the experimentally determined miscible polymer-polymer systems which are miscible because of specific interactions.

The only polydispersity that has received any significant theoretical or practical attention is that of molecular weight polydispersity. Koningsveld et al $(20,21)$ considered the effects of molecular weight polydispersity on polymer-solvent and polymerpolymer phase diagrams within the limitations of the Flory-Huggins theory (2-5). Among other things, Koningsveld (21) found that a polymer with molecular weight polydispersity 
has a critical point on a polymer-polymer phase diagram that is controlled by its z-average (equation 6 ) molecular weight

$$
M_{z}=\left(\Sigma w_{j} M_{j}^{2}\right) /\left(\Sigma w_{j} M_{j}\right)
$$

where $M_{z}$ is the $z$-average molecular weight of the polymer and $\Sigma w_{j}$ is the mass or weight of molecules with molecular weight $M_{j}$ in the sample.

In a consideration of the miscibility of block copolymers with homopolymers (or with random copolymers), the peculiarities of block copolymers must be considered. Most block copolymers that have been synthesized up to this time are phase-separated; blocks of each chemical type within the molecules form separate phases. The size of these separate phases is limited by the lengths of the blocks, thus, the phases are submolecular in dimension, generally with dimensions in at least one direction as small as 3 to $100 \mathrm{~nm}$. Therefore, one often speaks of microphase separation in block copolymers. In a microphase separated monodisperse block copolymer, the microphases may appear in a number of different morphologies (see Figure 1).

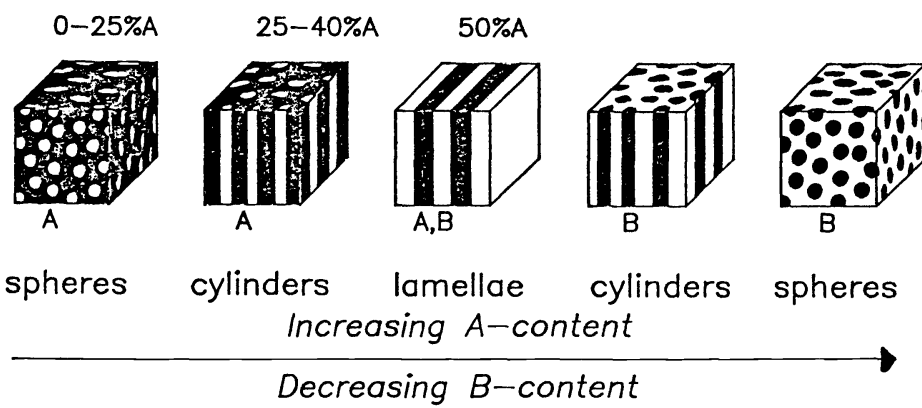

Figure 1. Equilibrium Morphologies of Block Copolymers

Figure 1 indicates that there is little volume within most of the block copolymer microphases to accommodate other polymer molecules; if another polymer dissolves in a microphase, the microphase must expand in at least one direction to accommodate the concomitant increase in volume. It is obvious that a microphase cannot increase in dimenions indefinitely because the blocks in the block copolymer cannot expand indefinitely. Therefore, only a limited amount of other polymer can dissolve in a microphase. There are other complications in a consideration of miscibility of other polymers into block copolymers. These will be considered briefly in terms of mixtures of binary block copolymers, those composed of just two chemically different monomers, $A$ and $B$, with one of the chemically identical homopolymers, poly-A. When present at very low concentration in poly-A, the $A-B$ block copolymer may migrate to the surface causing this surface to have the properties of poly-B, and/or the $A-B$ block copolymer may dissolve in the poly-A and, in addition, it may form micelles with poly-B block cores in the bulk of poly $A$. At higher concentrations of A-B block copolymer in the poly-A, the micelles of $A-B$ block copolymer may merge to form microphase separated regions as in Figure 1 ; these microphase separated arrays are sometimes called "mesophases." The poly-A regions of the "mesophases" may or may not contain admixed poly-A homopolymer; furthermore, several "mesophases", containing different percentages of admixed poly-A homopolymer, may be in equilibrium. A "mesophase" may be in equilibrium with a homogeneous phase containing $A-B$ block copolymer molecules dissolved in poly-A homopolymer. Two homogeneous phases containing different percentages of block copolymer may be in equilibrium. And so on. Whitmore and Noolandi (22) have used a mean field theory of inhomogeneous multicomponent polymeric systems (23) to calculate some very complex phase diagrams for this type of mixture. Similar complex phase diagrams were obtained experimentally for some mixtures of butadiene-styrene block copolymers with either polystyrene or polybutadiene by Roe and Zin (24). Three-component mixtures containing block copolymers are expected to present additional complications.

\section{EXPERIMENTAL DETERMINATION OF POLYMER-POLYMER MISCIBILITY AND PHASE DIAGRAMS}

The experimental study of polymer-polymer miscibility is complicated by the high viscosity of polymers, even when they are well above their glass transition temperatures. This means that two-phase systems, even when no block copolymers are involved, usually have one of the phases present as a dispersion in a ratrix of the other; it is generally impossible to collect the separate phases and determine their compositions by simple analysis as is usually done in solutions of low molecular weight substances. Worse yet, if the polymer mixture, or even one phase of the polymer mixture, is below its glass transi- 
tion temperature, the mobility of the polymer chains is so hindered that equilibrium is often not achieved. Therefore, the literature on polymer-polymer miscibility contains a number of ambiguous results, some of which have only recently been understood. For example, in my 1978 review (11), I cited the case of polystyrene-poly(vinyl methyl ether) mixtures, which had all the characteristics of a miscible polymer pair when cast as films from toluene solution but appeared completely immiscible when cast as films from trichloroethylene solution. It has since become clear that these two polymers are miscible at room temperature and that the appearance of incompatibility when cast fron trichloroethylene solution arises from the phase diagram of the three-component system of the two polymers with the solvent. An investigation of this polymer mixture in chloroform solution (25) showed that when the solvent concentration was high, the mixture was singlephase; as the solvent evaporated, the mixture entered a two-phase region on the phase diagram, and, on further evaporation of the solvent, the mixture probably underwent the glass transition in at least one of the phases before the mixture left this two-phase region to reenter a single-phase region. Thus, the mixture remains a tivo-phase system after all the solvent evaporates even though it should be a single-phase system at equilibrium. This is an example of a three-component phase diagram in which any set of two components is miscible at all concentrations, but, when all three components are mixed, phase separation may occur at some concentrations.

Polymer miscibility is usually studied by direct observation, either visual or microscopic, of the polymer sample, usually in the form of a thin film, or by observation of glass transitions and crystalline melting points in the samples. Miscible polymers that contain no crystalline phases form transparent films and exhibit no heterogeneity in a phase contrast microscope or in an electron microscope. Semicrystalline polymers cannot be studied by this method. Furthermore, immiscible fully amorphous polymers form transparent films when both polymers have the same refractive index or, when the refractive indices are different, they occasionally form two-layered films that appear transparent when a solution of the polymers is evaporated (26). The danger of using turbidity alone as a criterion of polymer miscibility is illustrated by the behavior of a mixture of chlorinated polyethylene, $27 \% \mathrm{Cl}$, with poly (methyl methacrylate). The sample appeared turbid below $70^{\circ} \mathrm{C}$, clear up to $80^{\circ} \mathrm{C}$, and then turbid again at higher temperatures (27). It was first assumed (27) that this mixture had an upper critical solution temperature around $70^{\circ} \mathrm{C}$, followed by a lower critical solution temperature around $80^{\circ} \mathrm{C}$, but later work (28) showed that the refractive indices of the two coexisting phases had different temperature coefficients and were coincidentally equal to each other in the 70 to $80^{\circ} \mathrm{C}$ interval.

A second way, often used, of studying polymer-polymer miscibility involves the observation of the glass transition temperatures of the amorphous phases in the mixtures. lliscible polymers should have only a single glass transition temperature; in the case of a mixture of block copolymer with homopolymer, if the homopolymer is miscible with only one of the microphases, then two glass transition temperatures should be observed: one for the unmixed microphase, and one for the mixed microphase. The single glass transition temperature criterion of polymer miscibility fails when the glass transition temperatures of the two polymers are very close to each other, too close to be resolved by the measuring techniques available. Glass transition temperatures of polymer mixtures have been measured by a variety of methods such as dilatometry, various dynamic mechanical methods, dielectric measurements, refractive-index versus temperature measurements, differential scanning calorimetry, differential thermal analysis, thermo-optical analysis, and radiothermoluminescence. In all these methods, the sample is observed over a large temperature range; problems may occur if the miscibility of the sample changes over this temperature range, especially at temperatures higher than the glass transition temperatures of all the phases in the sample. As a matter of fact, one way of observing the existence of a lower critical solution temperature at elevated temperatures in polymer mixtures has been the following. A sample that has exhibited a single glass transition temperature is heated to various temperatures above the highest glass transition temperature of any phase in the sample and is then quenched to well below room temperature from each elevated temperature in turn. After quenching from a temperature above the lolver critical solution temperature, two glass transition temperatures are observed in the sample. Lower critical solution temperatures in polymer-polymer mixtures are often observed in this way, as well as by the appearance of turbidity as the sample is heated.

Other methods for studying polymer-polymer miscibility exist, but they are either ambiguous or very recently developed and have not yet been used to test for the presence of one or more phases in previously untested systems. These methods will not be discussed here.

Upper critical solution temperatures in polymer-polymer mixtures are harder to observe than lower critical solution temperatures because the polymer mixture generally goes through the glass transition as the temperature is lowered. Thus, a polymer mixture that is miscible at some temperature above its glass transition temperature will have that single phase frozen-in as the sample is cooled below its glass transition tempera- 
ture. For this reason, upper critical solutions have been observed mostly in mixtures of oligomers, in which the molecules have a degree of polymerization so low that the glass transition temperatures are far below room temperature.

\section{SUMMARY OF LITERATURE DATA}

There were 282 chemically dissimilar polymer pairs in the literature that appeared to be miscible in the amorphous state at room temperature. Of these, approximately $75 \%$ appeared to be miscible because of specific interactions, mostly hydrogen-honding, and approxinately $15 \%$ appeared to be miscible because of a judicious choice (not necessarily a conscious one) of copolymer composition. Close to $40 \%$ of the 282 polymer pairs were semi-crystalline under the conditions in which they were investigated.

It was interesting to note that poly(2,6-dimethyl-1,4-phenylene oxide), which is known to be miscible with polystyrene, was also miscible with the styrene microphases in two block copoljmers of styrene and isoprene (29).

When a homopolymer is mixed with a random copolymer containing as one of its monomer units the same one that constitutes the homopolymer repeat unit, or when two random copolymers that have at least one monomer in common are mixed, miscibility over at least part of the composition range must be expected. Twenty-four such systems appeared in the literature, investigated over at least part of the composition range. Of particular interest are the thirteen random copolymers which have been investigated in terms of their miscibility when such copolymers, made up of chemically identical monomers but with each copolymer having a different average composition, are mixed. Equations 1,2, and 5 indicate why such copolymers should be immiscible when the compositions are sufficiently different. Table I summarizes the data found in the literature on the miscibility of these random copolymers. In Table I, DP refers to degree of polymerization. In fact, all the values in Table I should vary with degree of polymerization. The higher the degree of polymerization of the random copolymers, the lower the composition difference at which they become immiscible. The last copolymer in the Table, for example, remains miscible

Table I. Miscibility of random copolymers mde up of the same monomers when their compositions are different.

\begin{tabular}{|c|c|c|}
\hline Random Copolymer & $\begin{array}{l}\text { Miscible only when compo- } \\
\text { sition Difference } \leq\end{array}$ & Reference \\
\hline $\begin{array}{l}\text { Acrylonitrile-co-Butadiene } \\
\text { Acrylonitrile-co- } \alpha \text {-Methyl- } \\
\text { styrene-co-Styrene }\end{array}$ & $\begin{array}{l}22 \% \\
12 \%\end{array}$ & $\begin{array}{c}30-36 \\
37\end{array}$ \\
\hline $\begin{array}{l}\text { Acrylonitrile-co-Styrene } \\
\text { Butadiene-co-Styrene } \\
\text { Butyl Acrylate-co-Butyl } \\
\text { Methacrylate }\end{array}$ & $\begin{array}{l}3.5 \% \\
20 \% \\
25 \%\end{array}$ & $\begin{array}{c}38,39 \\
40-45 \\
46\end{array}$ \\
\hline $\begin{array}{l}\text { Butyl Acrylate-co-Ethyl } \\
\text { Acrylate }\end{array}$ & $25 \%$ & 46 \\
\hline $\begin{array}{l}\text { Butyl Acrylate-co-Methyl } \\
\text { Methacrylate }\end{array}$ & $10 \%$ & $47-48$ \\
\hline $\begin{array}{l}\text { Butyl Methacrylate-co-Methyl } \\
\text { Methacrylate }\end{array}$ & $20-30 \%$ depending on composition & 49 \\
\hline Chlorinated Ethylene & $\begin{array}{l}1.2-13.3 \text { wt } \% \mathrm{Cl} \text {, depending on } \\
\text { composition }\end{array}$ & 50,51 \\
\hline Chlorinated Vinyl Chloride & $\begin{array}{l}3-4 \% \mathrm{Cl} \text {, depending on composition } \\
\text { and on no. of } \mathrm{CCl}_{2} \text { groups }\end{array}$ & 52 \\
\hline $\begin{array}{l}\text { Ethyl Acrylate-co-Ethyl } \\
\text { Methacrylate }\end{array}$ & $50 \%$ & 46 \\
\hline $\begin{array}{l}\text { Ethyl Acrylate-co-Methyl } \\
\text { Methacrylate }\end{array}$ & $20-27 \%$ depending on composition & 49 \\
\hline $\begin{array}{l}\text { Methyl Acrylate-co-Methyl } \\
\text { Methacrylate }\end{array}$ & $\begin{array}{l}20-35 \% \text { depending on composition, } \\
D P=3000\end{array}$ & 49 \\
\hline
\end{tabular}

for composition differences between the two polymers from 23 to $48 \%$ when the degree of polymerization of the two polymers is 400 instead of 3000 (49). This is in accord with the predictions of Flory-Huggins theory. The change in miscibility with the average composition of the copolymers, however, is not predicted by the simple equations given in this paper. In fact, equation 5 is oversimplified; there are many reasons for this, one of which involves the effect of sequence distribution in the copolymers (53). 
There were ten polymer triads that formed single phase amorphous mixtures. In all but one case, these mixtures involved at least one random copolymer. In one case, a polymer tetrad, four different polymers, formed a single-phase mixture; this polymer tetrad was polystyrene, poly(2,6-dimethyl-1,4-phenylene oxide), poly(p-bromostyrene-costyrene), and poly(3-bromo-2, 6 -dimethyl-1,4-phenylene oxide-co-2,6-dimethyl-1,4-phenylene oxide) when both random copolymers contained no more than 26 mole \% brominated monomer.

The literature contained only nine examples of polymer pairs whose molecular weight dependence of miscibility was investigated. Seven of these polymer pairs had polystyrene as one component. Wata such as these are necessary to test available theoretical treatments of nolymer miscibility. It is interesting to note that no polymer pair whose miscibility depends on specific interactions between the polymers has been carefully investigated in terms of molecular weight dependence of miscibility. Such data, however, are probably necessary to help in the formulation of theoretical treatments suitable for such mixtures.

Eight polymer pairs appeared to have upper critical solution temperatures. On the other hand, there were fifty polymer pairs that appeared to have lower critical solution temperatures, often at temperatures above $100^{\circ} \mathrm{C}$. It was stated above that the observation of lower critical solution temperatures should be easier than the observation of upper critical solution temperatures.

\section{REFERENCES}

1. J. Erandrup and E. H. Immergut, eds. Polymer Handbook, 3rd ed., Hiley, New York, in press.

2. P. J. Flory, J. Chem. Phys. 9, 660 (1941).

3. P. 3. Flory, J. Chem. Phys. 10, 51 (1942).

4. M. L. Huggins, J. Chem. Phys. 9, 440 (1941).

5. H. L. Huggins, Ann. N.Y. Acad. Sci. 43, 1 (1942).

6. R. L. Scott, J. Chem. Phys. 17, 279 (1949).

7. H. Tompa, Trans. Faraday Soc. 45, 1142 (1949).

8. J. H. Hildebrand and R. L. Scott, "The Solubility of Nonelectrolytes," 3rd ed. Van Nostrand-Reinhold, Princeton, N.J., 1950; reprinted, Dover, New York, 1964.

9. J. H. Hildebrand and R. L. Scott, "Regular Solutions," Prentice-Hall, Englewood Cliffs, N.J., 1962.

10. J. H. Hildebrand, J. M. Prausnitz, and R. L. Scott, "Regular and Related Solutions," Van Nostrand Reinhold, New York, 1970.

11. S. Krause, Chapter 2 in Polymer Blends, Vol. 1, D. R. Paul and S. Mewman, eds., Academic, New York, 1978.

12. S. Krause, A. L. Smith, and M. G. Duden, J. Chem. Phys. 43, 2144 (1965).

13. G. ten Brinke, F. E. Karasz, and H. J. MacKnight, Macromolecules 16, 1827 (1983).

14. D. R. Paul and J. H. Barlow, Polymer 25, 487 (1984).

15. P. J. Flory, J. Am. Chem. Soc. 87, 1833 (1965).

16. L. P. Mcllaster, Macromolecules 6,760 (1973).

17. I. C. Sanchez, J. Macromol. Sci. Phys. B17, 565 (1980).

18. I. C. Sanchez and R. H. Lacombe, J. Phys. Chem. 80, 2352 (1976).

19. I. C. Sanchez and R. H. Lacombe, Macromolecules 11, 1145 (1978).

20. R. Koningsveld, H. A. G. Chermin and M. Gordon, Proc. Roy. Soc. A319, 331 (1970).

21. R. Koningsveld, Chem. Zuest. 26, 263 (1972).

22. M. D. Whitmore and J. Nool andi, Macromolecules 18, 2486 (1985).

23. K. H. Kong and J. Noolandi, Macromolecules 14, 727 (1981).

24. R.-J. Roe and W.-C. Zin, Macromolecules 17, 189 (1984).

25. A. Robard, D. Patterson, and G. Delmas, Macromolecules 10, 706 (1977).

26. L. J. Hughes and G. L. Brown, J. Appl. Polym. Sci. 5, 580 (1961).

27. D. J. Halsh, L. Shi, and Z. Chai, Polymer 22, 1005 (1981).

28. D. J. Halsh, J. S. Higgins, and Z. Chai, Polymer 23, 336 (1982).

29. G. C. Heyer and G. E. Tritscher, J. Appl. Polym. Sci. 22, 719 (1978).

30. K. Kosai and T. Higashino, Nippon Setchaku Kyokai Shi 11, 2 (1975).

31. G. M. Bartenev and G. S. Kongarov, Vysokomol. Soedin. 2, 1692 (1960); Rubber Chem. Technol. 36, 668 (1963).

32. P. J. Corish, Rubber Chem. Technol. 40, 324 (1967).

33. F. S. Cheng and J. L. Kardos, Polym. Prepr. Am. Chem. Soc. Div. Polym. Chem. 10, $615(1969)$.

34. A. H. Jorgensen, L. A. Chandler, and E. A. Collins, Rubber Chem. Technol. 46, 1087 (1973).

35. M. R. Ambler, J. Polym. Sci. Polym. Chem. Ed. 11, 1505 (1973).

36. L. A. Chandler and E. A. Collins, J. Appl. Polym. Sci. 13, 1585 (1969).

37. R. J. Slocombe, J. Polym. Sci. 26, 9 (1957).

38. G. E. Molau, J. Polym. Sci. Part B 3, 1007 (1965).

39. V. R. Landi, Rubber Chem. Technol. 45, 222 (1972).

40. D. I. Livingston and R. L. Rongone, Proc. Int. Rubber Technol. Conf., 5th, Brighton, llay (1967), p. 337 (Puh. 1968); Chem. Abstr. 72, 67965y (1970). 
41. G. L. Slonimskii and N. K. Komskaia, Zhur. Fiz. Khim. 30, 1746 (1956).

42. Y. F. Shutilin, Vysokomol. Soedin. Ser. B 23,780 (1981).

43. H. K. deDecker and D. J. Sabatine, Rubber Age 99(4), 73 (1967).

44. G. Kraus and K. W. Rollmann, Adv. Chem. Ser. 99, 189 (1971).

45. D. I. Livingston and J. E. Brown, Jr., Proc. 5th Int. Congr. Rheol. 1968 4, 25 (1970).

46. K. Kosai, T. Higashino, and N. Nishioka, Shikizai Kyokaishi 50, 76 (1977).

47. A. Dobry and F. Boyer-Kawenoki, J. Polym. Sci. 2, 90 (1947).

48. F. Kollinski and G. Markert, Adv. Chem. Ser. 99, 175 (1971).

49. F. Kollinsky and G. Markert, Makromol. Chem. 121, 117 (1969).

50. H. J. CSivald and E. T. Kubu, SPE Trans. 3, 168 (1963).

51. Z. Chai and R. Sun, Polymer 24, 1279 (1983).

52. H. H. Lehr, Polym. Eng. Sci. 25, 1056 (1985).

53. A. C. Balazs, F. E. Karasz, W. J. MacKnight, H. Ueda, and I. C. Sanchez, Macromolecules 18, $2784(1985)$. 